\title{
Boundary-layer Type Solutions for Initial Platelet Activation and Deposition
}

\author{
T. DAVID ${ }^{\mathrm{a}, *}$, P.G. DE GROOT ${ }^{\mathrm{b}}$ and P.G. WALKER ${ }^{\mathrm{a}}$ \\ ${ }^{\mathrm{a}}$ Department of Mechanical Engineering, University of Canterbury, Private Bag 4800, Christchurch, New Zealand; ${ }^{\mathrm{b}}$ Department of Haematology, \\ University Hospital Utrecht, Utrecht, The Netherlands
}

(Received 7 April 2000; Revised 22 January 2002; In final form 25 April 2002)

\begin{abstract}
This paper presents, on the basis of high Peclet number, a mathematical model for the activation and initial adhesion of flowing platelets onto a surface. In contrast to past work, the model is applicable to general 2D and axi-symmetric flows where the wall shear stress is known a priori. Results indicate that for high activation reaction rates there exist two layers, one containing only activated platelets and the other both activated and non-activated platelets. Fundamental relationships are proposed between the adhesion rate of platelets to the surface and the characteristic parameters of Peclet number and Reynolds number. Activation in the bulk fluid (blood) is characterised by the Damkohler number, which is a function of activation rate and the free-stream velocity. It is shown that, as the free-stream velocity varies, there exists a maximum of activated platelet flux to the wall for particular values of the velocity. These values, at which the maximum occur, are themselves functions of the platelet activation rate. As the free-stream velocity increases the activation of platelets ceases altogether and adhesion is reduced to a very small value strengthening the hypothesis of the correlation between atherogenesis/thrombogenesis and areas of low shear.
\end{abstract}

Keywords: Platelet adhesion; Boundary layer flow

\section{INTRODUCTION}

Atherosclerosis is one of the leading causes of death in the world today. In the United States alone, in 1995, there were nearly half a million deaths attributable to coronary disease (Wilson and Ferguson, 1999). It is now reasonably well established that there exists a strong relationship between the flow of blood, the permeability of the endothelium to cholesterol and other LDLs (lowdensity lipoproteins) leading to the formation of atherosclerotic plaques. Platelet adhesion and subsequent aggregation are also important in atherogenesis, along with endothelial dysfunction and atherosclerotic plaque instability. In addition, the first stage of thrombogenesis is platelet adhesion on a surface followed by aggregation and the formation of platelet mural thrombi (Friedman and Leonard, 1971). The resulting thrombosis and/or embolisation from diseased arteries produces a wide variety of clinical scenarios; myocardial infarction, strokes and gangrene. Vascular geometry can now be regarded as a risk factor due to the influence of that geometry on local haemodynamic effects. In particular, it is extremely important to gain a thorough understanding of the relationship between vascular geometry, blood flow and the onset of thrombus formation in both the natural and diseased arteries. Although the formation of thrombi and platelet activation in stasis is fairly well understood, the influence of blood-flow characteristics has yet to be fully investigated. Fluid dynamic studies of blood flow, in models of arteries, suggest a set of fluid dynamic conditions that appear to predispose thrombus formation (platelet adhesion), principally at arterial bifurcations, T-junctions and curved sections.

Over the past 20 years, there have been a number of proposed models for platelet adhesion and its relationship

*Corresponding author. E-mail: t.david@mech.canterbury.ac.nz 
to blood flow and vascular geometry (Leonard et al., 1972; Turitto and Baumgartner, 1975; Kratzer and Kinder, 1986; Affeld et al., 1995) as well as a considerable amount of experimental data collected (Petschek et al., 1968; Friedman and Leonard, 1971; Leonard et al., 1972; Turitto and Baumgartner, 1975; Kratzer and Kinder, 1986; Strong et al., 1987; Tippe et al., 1992; Reininger et al., 1993; Schoephoerster et al., 1993; Affeld et al., 1995; Reininger et al., 1996). The models concentrated on both stagnation point flow as well as simple linear shear flow. However, initially it was assumed that a diffusioncontrolled system existed where the adhesion rate was large compared to the diffusion coefficient. Recently, workers have begun to call into question the diffusioncontrolled system and look more closely at intermediate kinetics. Wall shear stress has been proposed as a possible mediator for platelet adhesion (Rajagopalan et al., 1988; Weiss, 1995).

Experiments have been done in parallel plate flow chambers, annular expansion tubes (Turitto and Baumgartner, 1975; Karino and Goldsmith, 1979) and in stagnation flow regimes. However, the main theoretical analysis has been attempted (Turitto and Baumgartner, 1975; Strong et al., 1987) only for the parallel flow system, where wall shear rate is constant. Numerical models have been developed that include the integration of computational fluid dynamics with relatively complex kinetic mechanisms (Sorensen et al., 1999a,b). However, the flow regime was of constant shear type and the presented results concentrated on obtaining reaction-rate parameters from comparison with experiment, although this model could very well be used for more complex flow conditions. Affeld et al. (1995) investigated platelet adhesion in a stagnation point flow chamber. The results from the experiment indicated, as had others, that the small neighbourhood of the stagnation point streamline was devoid of platelets along with the existence of a domain of maximum platelet adhesion just downstream of the stagnation point streamline. They put forward the hypothesis that thrombin emanating from the adhered platelet granules would be convected downstream and activate other platelets flowing above the adhesion surface and that the maximum adhesion rate occurred for a "critical wall shear rate".

The presented work puts forward a boundary-layer type model for platelet activation and adhesion. This model is applicable to axi-symmetric flows, commonly found in the neighbourhoods of stagnation points and 2D flows where, in both cases, the wall shear stress is known a priori. It covers a large range of kinetic rates, from reaction controlled through intermediate kinetics to diffusioncontrolled systems. We investigate the role of thrombin, emanating from adhered platelets on the activation of flowing non-activated platelets and we compare the results with experiment. The model provides a parametric representation of platelet activation and adhesion through the three fundamental parameters of Peclet, Reynolds and Damkohler numbers and indicates a fundamental relationship between adhesion rate and the roles of convection and diffusion.

\section{MATHEMATICAL MODEL}

In order to fully understand the mechanism of thrombogenesis, it is important to investigate the chemicals important to platelet activation. One proposed mechanism (Hubbell and McIntire, 1986) is that adherent platelets, if sufficiently activated, produce local high concentrations of platelet activating substances, which then diffuse and convect into the flow thereby activating platelets in the bulk flow. These highly reactant chemicals are important in determining the complex chemical mechanisms, which make up the clotting cascade; they all essentially diffuse outward from the activated and adhered platelet. This may then activate other platelets that may be convected and/or diffused into the reaction neighbourhood.

Three chemicals have been identified as playing important roles in platelet activation;

Adenosine diphosphate: released from the dense granules of the platelet.

Thromboxane A2: enzymatically generated on or near the membrane of the platelet.

Thrombin: secreted from platelets both when activated and adhering.

From a modelling viewpoint, we may simplify this system somewhat by lumping these chemicals into a single species, which for the purpose of clarity, shall be denoted as thrombin. Both activated and non-activated platelets can adhere to the wall or surface although this will be at different rates.

\section{Basic Theory}

In this section, we set out the basic conservation equations for both fluid flow and reactive species. In order to compare with other workers, we assume that firstly blood be modelled as a Newtonian fluid, secondly that the system is of constant density and temperature and finally, although physiological blood flows are pulsatile in nature, we assume a steady-state system. The steady-state conservation of mass and momentum in vector form is written as

$$
\nabla \cdot \mathbf{u}=0
$$

and

$$
\rho\left(\nabla\left(\frac{\mathbf{u}^{2}}{2}\right)-\mathbf{u} \times(\nabla \times \mathbf{u})\right)=-\nabla p+\mu \nabla^{2} \mathbf{u}
$$

Here $\mathbf{u}$ is the velocity vector in a general orthogonal set of co-ordinates, $\rho$ is the density, $\mu$ is the dynamic viscosity and $p$ is the pressure that varies due to dynamic variations 
in fluid velocity alone. Red blood cells (RBC) have a considerable effect on the diffusion of platelets, an effect that is enhanced by shear rate. However, the main experimental evidence with which we compare our analysis was done with platelet-rich plasma containing no RBCs (Affeld et al., 1995). Hence for the present we assume constant diffusion coefficients.

Although adhesion is a time-dependent process for initial adhesion Strong et al. (1987) have shown that for small times adhesion is the product of a constant rate of adhesion and time. We can assume steady state and then use a simple product of platelet flux and small time to evaluate the number of platelets adhering. Hence, for this present model, we look at steady state only (the similarity solution method is not restricted to steady-state methods see David et al., 2001a). A general conservation equation for the $i$ th cellular/chemical species can be written, again in vector form, as

$$
\nabla \cdot\left(\mathbf{u} \phi_{i}\right)=\nabla \cdot\left(D_{i} \nabla \phi_{i}\right)+W_{i} \quad i=1, \ldots, N
$$

$\phi_{i}$ is the $i$ th mass fraction defined as $\phi_{i}=\rho_{i} / \rho, W_{i}$ is the rate of production of the $i$ th species mass fraction and $D_{i}$ is the $i$ th species diffusion coefficient assuming a Fickian diffusion model. Since the bulk fluid density is constant, the momentum and species-conservation equations are effectively de-coupled.

The diffusion coefficient for platelets may be determined by the use of the Stokes-Einstein equation

$$
D_{\mathrm{pl}}=\frac{B T}{6 \pi \mu r_{\mathrm{pl}}}
$$

Here $B$ is the Boltzmann constant, $T$ is the absolute temperature, $\mu$ is the coefficient of viscosity of plasma and $r_{\mathrm{pl}}$ is the radius of a platelet. Using the above and an assumption of a core-body temperature of $37^{\circ}$ for $T, D_{\mathrm{pl}}$, the platelet diffusion coefficient was calculated as $1.7 \times 10^{-13} \mathrm{~m}^{2} \mathrm{~s}^{-1}$. This compares favourably with values calculated by many other workers (Strong et al., 1987). If RBC augmentation is modelled, then this diffusion coefficient value can increase by as much as two orders of magnitude (Strong et al., 1987), depending on the shear rate.

For the case presented here, it was assumed that platelet activation occurred in the bulk fluid, through a reaction with thrombin, and that the phenomenon of platelet adhesion to the wall is represented by a simple reaction boundary condition, where platelets are either "free" or permanently adhered. Once adhered, it is assumed that thrombin is diffused outward from the adhered platelet at a specified rate. The thrombin emanates from the internal part of the cell and it has been shown that the time taken to diffuse out is quite long due to the canalicular structure of the platelet membrane (Fogelson and Wang, 1996). For this model, we can assume that although the platelet may be activated in the bulk fluid, it does not release thrombin until adhered at the surface. In addition, it is assumed that no reversible reactions occur representing the worst-case scenario for thrombogenesis. The reaction mechanisms are given by

thrombin + platelets $\stackrel{k_{\mathrm{b}}}{\rightarrow}$ activated platelets + thrombin

surface + activated platelets $\stackrel{k_{\mathrm{wa}}}{\rightarrow}$ adhered platelets + surface

surface + platelets $\stackrel{k_{\mathrm{wp}}}{\rightarrow}$ adhered platelets + surface

adhered platelets $\stackrel{k_{\mathrm{wt}}}{\longrightarrow}$ adhered platelets + thrombin

Here $k_{\mathrm{b}}$, is the forward rate for the bulk reaction and $k_{\mathrm{wa}}$, $k_{\mathrm{wp}}$ and $k_{\mathrm{wt}}$ are surface (or wall) reactions for activated platelets, non-activated platelets and thrombin, respectively. The constraint that thrombin is effused from an activated platelet only when it has adhered can be relaxed so that the first reaction mechanism given in Eq. (5) can be rewritten as

$$
\begin{aligned}
\text { thrombin }+ \text { platelets } \stackrel{k_{b}}{\longrightarrow} \text { activated platelets } \\
\\
+\alpha \text { thrombin }
\end{aligned}
$$

where $\alpha>1$; this essentially corresponds to a non-zero source term in the conservation equation for thrombin. We assume that the both platelets and activated platelets become adhered at the surface at a rate proportional to their concentration at the wall in a similar manner to that given by Sorensen et al. (1999b). We can write these surface boundary conditions as

$$
\begin{gathered}
\left.D_{\text {act pl }} \frac{\partial \phi_{\text {act pl }}}{\partial n}\right|_{\text {surface }}=\left.k_{\text {wa }} \phi_{\text {act pl }}\right|_{\text {surface }} \\
\left.D_{\mathrm{pl}} \frac{\partial \phi_{\mathrm{pl}}}{\partial n}\right|_{\text {surface }}=\left.k_{\mathrm{wp}} \phi_{\mathrm{pl}}\right|_{\text {surface }}
\end{gathered}
$$

Here $n$ is the co-ordinate normal to the reacting surface. Finally, the production of thrombin is determined by the rate of adhesion of platelets. So that in a similar manner we can write

$$
\left.D_{\mathrm{th}} \frac{\partial \phi_{\mathrm{th}}}{\partial n}\right|_{\text {surface }}=-k_{\mathrm{wt}}\left[k_{\mathrm{wa}} \phi_{\mathrm{act} \mathrm{pl}}+k_{\mathrm{wp}} \phi_{\mathrm{pl}}\right]
$$

The modelling procedure has been split into two distinct areas, an analytical solution for the limiting case of infinite Damkohler number and a numerical procedure for the general coupled equations of species conservation.

The analysis is applicable to both axi-symmetric and 2D flows and Fig. 1(a) and (b) shows the co-ordinate systems for both cases

For clarity the fluid domain will be assumed to be of an axi-symmetric form, modelling the stagnation point flow. However, this analysis may be used for any a priori known 


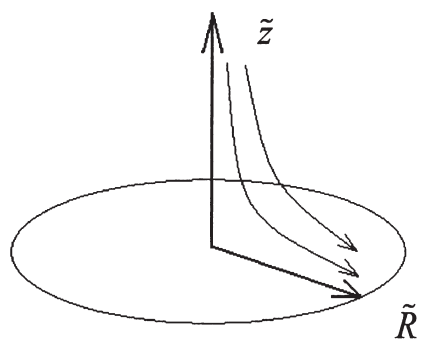

(a)

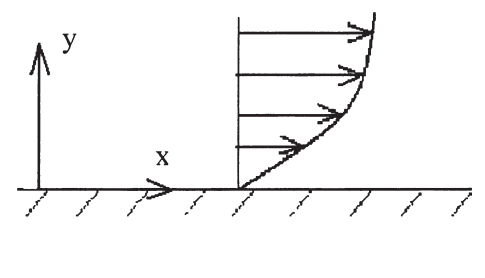

(b)

FIGURE 1 (a) Co-ordinate system for stagnation point flow. (b) Co-ordinate system for parallel plate flow.

2D velocity field where the boundary-layer flow does not detach. We concentrate on phenomena in the viscous boundary layer and the mass-transfer sub-layer and as such use a boundary-layer formulation. As a first step in the solution process, we assume that mass diffusion in the stream-wise radial $(\tilde{R})$ direction is negligible compared to that in the axial ( $\tilde{z})$ direction. With these assumptions, the constant density conservation equation for the mass fraction of the $i$ th cellular species, $\phi_{i}$, can be written in the boundary-layer type form (Schlichting, 1960) as

$$
\tilde{u} \frac{\partial \tilde{\phi}_{i}}{\partial \tilde{R}}+\tilde{v} \frac{\partial \tilde{\phi}_{i}}{\partial \tilde{z}}=\tilde{D}_{i} \frac{\partial^{2} \tilde{\phi}_{i}}{\partial \tilde{z}^{2}}+\tilde{W}_{i} \quad i=1, \ldots, N
$$

where $\tilde{u}$ and $\tilde{v}$ are the velocity components for the $\tilde{R}$ and $\tilde{z}$ directions, respectively, and $N$ is the total number of species participating within the domain. Again, in a similar manner to Sorensen et al. (1999b), $W_{i}$ can be modelled as a first-order reaction mechanism so that

$$
\tilde{W}_{i}=k_{\mathrm{ib}}\left[\phi_{k}\right]\left[\phi_{1}\right]
$$

where the associated reaction is given by

$$
\text { species } k+\operatorname{species} \ell \stackrel{k_{\mathrm{ib}}}{\rightarrow} \text { species } i
$$

By choosing appropriate length, velocity and concentration scales, $L, U_{\infty}$ and $\phi_{\mathrm{pl} \infty}$, respectively, such that

$$
\begin{gathered}
u=\frac{\tilde{u}}{U_{\infty}} ; v=\frac{\tilde{v}}{U_{\infty}} ; R=\frac{\tilde{R}}{L} ; z=\frac{\tilde{z}}{L} \\
\phi_{i}=\frac{\tilde{\phi}_{i}}{\phi_{\mathrm{pl} \infty}}
\end{gathered}
$$

the non-dimensionalised form of the species equation is given as

$$
u \frac{\partial \phi_{i}}{\partial R}+v \frac{\partial \phi_{i}}{\partial z}=\frac{D_{i}}{P e} \frac{\partial^{2} \phi_{i}}{\partial z^{2}}+W_{i} \quad i=\mathrm{pl}, \text { th, act } \mathrm{pl}
$$

where $P e$, is the Peclet number defined as

$$
P e=\frac{U_{\infty} L}{\tilde{D}_{\mathrm{pl}}}
$$

the non-dimensional diffusion coefficient $D_{i}$ is defined as

$$
D_{i}=\frac{\tilde{D}_{i}}{\tilde{D}_{\mathrm{pl}}}
$$

If only a single bulk reaction is modelled, then the nondimensional production rates for platelets, activated platelets and thrombin are given as

$$
\begin{aligned}
W_{\mathrm{pl}} & =\frac{\tilde{W}_{\mathrm{pl}} L}{U_{\infty}}=\frac{k_{\mathrm{b}} L}{U_{\infty}}\left[\phi_{\mathrm{pl}}\right]\left[\phi_{\mathrm{th}}\right]=\operatorname{Dm}\left[\phi_{\mathrm{pl}}\right]\left[\phi_{\mathrm{th}}\right] \\
W_{\mathrm{actpl}} & =\frac{\tilde{W}_{\mathrm{actpl}} L}{U_{\infty}}=-\operatorname{Dm}\left[\phi_{\mathrm{pl}}\right]\left[\phi_{\mathrm{th}}\right] \\
W_{\mathrm{th}} & =\frac{\tilde{W}_{\mathrm{th}} L}{U_{\infty}}=0
\end{aligned}
$$

$D m$ is the Damkohler number, a ratio of fluid transit time to chemical reaction time. The diffusion coefficients for cells moving in blood plasma are extremely low and, thus, $\mathrm{Pe}$ is correspondingly high. For physiological conditions, the velocity boundary-layer thickness, $\delta \sim R e^{1 / 2}$, is large compared to the species mass-transfer boundary layer, $\varepsilon \sim P e^{1 / 2}$ as shown in Fig. 2. The velocity profile can, therefore, be assumed to have a linear form and the velocity vector field may be evaluated independently.

For the cases presented here, the velocity field is assumed known a priori and the wall shear stress evaluated from this. For the particular case of a stagnation point flow, the non-dimensional wall shear stress function, $\tau_{\mathrm{w}}(R)$, may be analytically generated using the Heimenz solution (Schlichting, 1960). However, this solution is only valid in

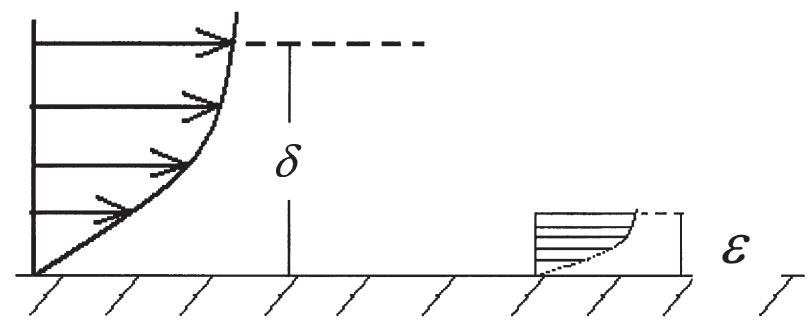

FIGURE 2 Comparison and characteristic depths of the boundary layer for momentum, $\delta$, and mass transfer, $\varepsilon$. 


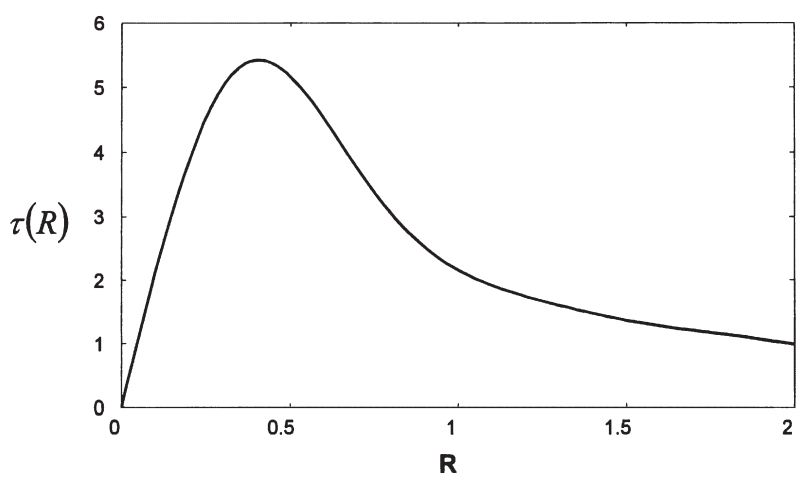

FIGURE 3 Wall shear stress, $\tau_{\mathrm{w}}(R)$ as a function of non-dimensional radial co-ordinate for the axi-symmetric stagnation point flow.

a small neighbourhood of the stagnation streamline where the shear stress is a linear function of the radial coordinate, $R$. It was felt important, especially when comparing with the results of Affeld et al. (1995), that a much wider domain should be considered. Hence $\tau_{\mathrm{w}}(R)$ was derived from a numerical solution to the NavierStokes equations (David et al., 2001b). The computational domain mimicked the experiment by Affeld et al., (1995), with no slip boundary conditions on the lower and upper walls, a specified parabolic profile at the pipe entrance (positioned at right-angles to the adherent surface) and a zero gradient condition at the outlet. In the interests of brevity, we refer to the work of David et al. (2001b) for a description of the computational domain. Figure 3 shows the wall shear stress as a function of the radial co-ordinate, $R$. This compares very well with that given by Affeld et al. (1995) as shown by Thomas (2000).

Results for a single species model (David et al., 2001b) have shown that for high $P e$ with the stream-function definition given by

$$
\psi=\frac{1}{2} \tau_{\mathrm{w}}(R) R e z^{2}
$$

and an appropriate von Mises transformation (Schlichting, 1960) a similarity variable, $\eta$, defined by

$$
\eta=z \frac{\left\{\tau_{\mathrm{w}}(R)\right\}^{1 / 2}(P e R e)^{1 / 3}}{\left[9 \int_{0}^{R}\left\{\tau_{\mathrm{w}}(\gamma)\right\}^{1 / 2} \mathrm{~d} \gamma\right]^{1 / 3}}=z \beta(R)
$$

may be used, where the non-dimensional wall shear stress is given by

$$
\tau_{\mathrm{w}}(R)=\frac{\tilde{\tau}_{\mathrm{w}}(\tilde{R})}{\rho U_{\infty}^{2}}
$$

and the Reynolds number $R e$ is defined as

$$
R e=\frac{\rho U_{\infty} L}{\mu}
$$

A full derivation of the similarity variable is given in the appendix. We note that close to the stagnation point streamline the shear stress is a linear function of the radial coordinate, $\tau_{\mathrm{w}}(R)=a R$, say. Substitute this form into Eq. (12) then it is easy to show that

$$
\lim _{R \rightarrow 0}[\beta(R)]=\left(\frac{a \operatorname{RePe}}{6}\right)^{1 / 3}
$$

So that even though the wall shear stress tends to zero at the stagnation point, the similarity variable is defined at $R=0$. In comparison with other workers where the flow in the Cartesian co-ordinate system is of a Poiseuille type, then $\tau_{\mathrm{w}}(x)$ is of a constant value and the similarity variable has the form given by

$$
\eta=\left(\frac{\operatorname{RePe} \tau_{\mathrm{w}}}{9\left(x-x_{0}\right)}\right)^{1 / 3} y
$$

with $\tau_{\mathrm{w}}(x)$, the non-dimensional constant wall shears stress in the stream-wise direction $x$. Using either Eq. (12) or (14) to define the similarity variable depending on whether a cylindrical or Cartesian co-ordinate system is considered. In both cases, the non-dimensional species conservation equation becomes for the $i$ th species mass fraction

$$
\begin{aligned}
D_{i} \frac{\mathrm{d}^{2} \phi_{i}}{\mathrm{~d} \eta^{2}}+3 \eta^{2} \frac{\mathrm{d} \phi_{i}}{\mathrm{~d} \eta} & =\mathfrak{M}\left[\phi_{k}\right]\left[\phi_{1}\right] \\
\mathfrak{M} & =D m \frac{P e}{\beta^{2}(R)} .
\end{aligned}
$$

The resulting ordinary differential equations can be used in a variety of fluid flow cases where $\tau_{\mathrm{w}}(R)$ is known $a$ priori and is well behaved. The flux boundary condition at the surface is of the form (for the cylindrical case)

$$
\begin{aligned}
\frac{\partial \phi_{i}}{\partial z} & =\frac{\mathrm{d} \phi_{i}}{\mathrm{~d} \eta} \frac{\partial \eta}{\partial z}=\frac{\mathrm{d} \phi_{i}}{\mathrm{~d} \eta} \beta(R)=\kappa_{i} \phi_{i \text { wall }} \\
\kappa_{i} & =\frac{k_{i} L}{\phi_{\mathrm{pl} \infty} \tilde{D}_{i}}
\end{aligned}
$$

Here $\kappa_{i}$ may be thought of as a Sherwood number for the $i$ th species. However, since only a single reaction occurs in the bulk fluid, the Damkohler numbers $D m_{i}$ can be considered to be equal for all $i, D m_{i}=D m, \forall_{i}=\mathrm{pl}$, th. Finally, a boundary condition modelling the species mass fraction at a large distance away from the surface is needed. We assume that far away from the adhesion surface the concentration of species is a constant. So that

$$
\eta \rightarrow \infty ; \quad \phi_{i}(\eta) \rightarrow \phi_{i \infty}
$$

We note that there are two limiting conditions to the problem. Firstly, where the Damkohler number is small and secondly, where it is large. It is normally the case that the activation of platelets is fast and hence the Damkohler number is large for values of $L / U_{\infty} \sim O(1)$. 
TABLE I $0 \leq \eta<\eta^{*}$

\begin{tabular}{llll}
\hline Platelets & \multicolumn{1}{c}{ Activated platelets } & Thrombin \\
\hline$\eta=0 ; \quad \frac{\partial \phi_{\mathrm{pl}}}{\partial \eta}=\frac{\kappa_{\mathrm{wp}}}{\beta(R)} \phi_{\mathrm{pl}}$ & $\eta=0 ; \quad \frac{\partial \phi_{\mathrm{actpl}}=\frac{\kappa_{\mathrm{wa}}}{\beta \eta} \phi_{\mathrm{actpl}}}{\partial(R)}$ & $\eta=0 ; \quad \frac{\partial \phi_{\mathrm{ll}}}{\partial \eta}=-\frac{\kappa_{\mathrm{wl}}}{\beta(R)}\left[\kappa_{\mathrm{wa}} \phi_{\mathrm{act} \mathrm{pl}}+\kappa_{\mathrm{wp}} \phi_{\mathrm{pl}}\right]$ \\
$\eta=\eta^{*} ; \quad \phi_{\mathrm{pl}}=0.0$ & $\eta=\eta^{*} ; \quad \phi_{\mathrm{actpl}}=1.0$ & \\
\hline
\end{tabular}

Large Damkohler number cannot occur for negligibly small $U_{\infty}$, since this would invalidate the similarity variable. We investigate first the case of $D m \rightarrow \infty$ where we obtain an analytical solution and then use a numerical procedure for the case for intermediate values of Dm. The analytical and numerical solutions are then compared.

\section{Analytical Solution for Dm $\rightarrow \infty$}

For this case, the domain of reaction, where the activation of platelets by thrombin takes place, is an infinitely small sheet. Let us assume that the position of this sheet is $\eta^{*}$. The concentration of non-activated platelets at this position is zero and all platelets have been activated. The set of ordinary differential equations for platelets, activated platelets and thrombin become

$$
D_{i} \frac{\mathrm{d}^{2} \phi_{i}}{\mathrm{~d} \eta^{2}}+3 \eta^{2} \frac{\mathrm{d} \phi_{i}}{\mathrm{~d} \eta}=0 \quad i=1, \ldots 3
$$

The RHS of Eq. (19) is zero since all the reaction terms are now embedded in a infinitesimally thin reaction sheet and the equation is satisfied in the entire domain excluding the point $\eta=\eta^{*}$. The domain is split into two sections $0 \leq \eta<\eta^{*}$ and $\eta^{*}<\eta<\infty$. The boundary conditions are set out in Tables I and II.

To close the system, we have to find the value of $\eta *$. Since the convective velocity for reacting species at the reaction surface is the same, then thrombin and platelets can only approach each other by a diffusive process and only in stoichiometric proportions. This means that the diffusive mass flux must be in stoichiometric proportions, thus

$$
\left.m_{\mathrm{th}} \frac{\mathrm{d} \phi_{\mathrm{th}}}{\mathrm{d} \eta}\right|_{\eta=\eta^{*}}=-\left.m_{\mathrm{pl}} \frac{\mathrm{d} \phi_{\mathrm{pl}}}{\mathrm{d} \eta}\right|_{\eta=\eta^{*}}
$$

where $m_{\mathrm{th}}$ and $m_{\mathrm{pl}}$ are the molecular masses of thrombin and platelets, respectively.

TABLE II $\quad \eta^{*} \leq \eta<\infty \quad(21)$

\begin{tabular}{lll}
\hline Platelets & Activated platelets & Thrombin \\
\hline$\eta=\eta^{*} ; \phi_{\mathrm{pl}}=0.0$ & $\eta=\eta^{*} ; \phi_{\text {act } \mathrm{pl}}=1.0$ & $\eta \rightarrow \infty ; \quad \phi_{\mathrm{th}} \rightarrow 0.0$ \\
$\eta \rightarrow \infty ; \phi_{\mathrm{pl}} \rightarrow 1.0$ & $\eta \rightarrow \infty ; \quad \phi_{\text {act pl }} \rightarrow 0.0$ & \\
\hline
\end{tabular}

We are now in a position to solve the resulting equations (19) subject to the boundary conditions (18) and (21). For the individual domains the solutions are

$\boldsymbol{0} \leq \boldsymbol{\eta}<\boldsymbol{\eta} *$

Platelets

$$
\phi_{\mathrm{pl}}=0
$$

Activated platelets

$$
\begin{aligned}
\phi_{\operatorname{actpl}}(\eta)= & \left(\frac{3 \kappa_{\mathrm{wa}}}{3 D_{\mathrm{actpl}} \beta(R)+\kappa_{\mathrm{wa}} \Gamma\left(1 / 3, \eta^{*}\right)}\right) \\
& \times \int_{0}^{\eta} \exp \left(-\gamma^{3}\right) \mathrm{d} \gamma \\
& +\frac{3 D_{\mathrm{act} p l} \beta(R)}{3 D_{\mathrm{actpl}} \beta(R)+\kappa_{\mathrm{wa}} \Gamma\left(1 / 3, \eta^{*}\right)}
\end{aligned}
$$

Thrombin

$$
\begin{aligned}
\phi_{\mathrm{th}}(\eta)= & \frac{-3 \kappa_{\mathrm{wt}}}{3 D_{\mathrm{Act} \mathrm{pl}} \beta(R)+\kappa_{\mathrm{wp}} \Gamma\left(1 / 3, \eta^{*}\right)} \\
& \times\left(\int_{0}^{\eta} \exp \left(-\frac{\gamma^{3}}{D_{\mathrm{th}}}\right) \mathrm{d} \gamma-\frac{D_{\mathrm{th}}^{1 / 3} \Gamma(1 / 3, \infty)}{3}\right)
\end{aligned}
$$

$\boldsymbol{\eta}^{*} \leq \boldsymbol{\eta}<\infty$

Platelets

$$
\begin{aligned}
\phi_{\mathrm{pl}}(\eta)= & \frac{3 \phi_{\mathrm{pl} \infty}}{\Gamma(1 / 3, \infty)-\Gamma\left(1 / 3, \eta^{*}\right)} \\
& \times\left(\int_{0}^{\eta} \exp \left(-\gamma^{3}\right) \mathrm{d} \gamma-\frac{\Gamma\left(1 / 3, \eta^{*}\right)}{3}\right)
\end{aligned}
$$

Activated platelets

$$
\begin{aligned}
\phi_{\mathrm{actpl}}(\eta)= & \frac{3 \phi_{\mathrm{pl} \infty}}{\Gamma\left(1 / 3, \eta^{*}\right)-\Gamma(1 / 3, \infty)} \\
& \times\left(\int_{0}^{\eta} \exp \left(-\gamma^{3}\right) \mathrm{d} \gamma-\frac{\Gamma(1 / 3, \infty)}{3}\right)
\end{aligned}
$$


Thrombin

$$
\begin{aligned}
\phi_{\mathrm{th}}(\eta)= & \frac{-3 \kappa_{\mathrm{wt}}}{3 D_{\mathrm{act} \mathrm{pl}} \beta(R)+\kappa_{\mathrm{wp}} \Gamma\left(1 / 3, \eta^{*}\right)} \\
& \times\left(\int_{0}^{\eta} \exp \left(-\frac{\gamma^{3}}{D_{\mathrm{th}}}\right) \mathrm{d} \gamma-\frac{D_{\mathrm{th}}^{\frac{1}{3}} \Gamma(1 / 3, \infty)}{3}\right)
\end{aligned}
$$

Here, $\Gamma(a, \chi)=\int_{0}^{\chi} e^{-t} t^{a-1} \mathrm{~d} t$ is the incomplete gamma function. The concentrations are continuous across the reaction sheet; however, the derivatives are not, except that for thrombin where all its derivatives are continuous. This is due to the fact that thrombin is essentially a catalyst in the reaction. To complete the analysis for large Damkohler number we use the stoichiometric Eq. (20) for the fluxes of thrombin and platelets and substitute in the derivatives of the known solutions (evaluated as $\eta \rightarrow \eta_{ \pm}^{*}$ ) and the masses for thrombin and platelets. To find $\eta^{*}$, we require the root of the following equation

$$
\begin{aligned}
& \frac{m_{\mathrm{th}}}{m_{\mathrm{pl}}} \kappa_{\mathrm{wa}} \exp \left(\frac{-\eta^{*}}{D_{\mathrm{th}}}\right)\left[\Gamma\left(\frac{1}{3}, \infty\right)-\Gamma\left(\frac{1}{3}, \eta^{*}\right)\right] \\
& -\exp \left(-\eta^{*}\right)\left[3 \beta(R)+\kappa_{\mathrm{wp}} \Gamma\left(\frac{1}{3}, \eta^{*}\right)\right]=0
\end{aligned}
$$

The above equation is solved using a Maple ${ }^{\mathrm{TM}}$ root finding algorithm for specific values of the molecular masses and the surface reaction rates.

\section{Numerical Solution for Intermediate Dm}

For the case where the Damkohler number is neither large nor small, we use a numerical solution method. The domain is treated as a continuous range and here the set of differential equations governing the concentration of species are written as

$$
\begin{aligned}
& D_{\mathrm{pl}} \frac{\mathrm{d}^{2} \phi_{\mathrm{pl}}}{\mathrm{d} \eta^{2}}+3 \eta^{2} \frac{\mathrm{d} \phi_{\mathrm{pl}}}{\mathrm{d} \eta}=-\mathfrak{M}_{\mathrm{pl}}\left[\phi_{\mathrm{th}}\right]\left[\phi_{\mathrm{pl}}\right] \\
& D_{\mathrm{act} \mathrm{pl}} \frac{\mathrm{d}^{2} \phi_{\mathrm{act} \mathrm{pl}}}{\mathrm{d} \eta^{2}}+3 \eta^{2} \frac{\mathrm{d} \phi_{\mathrm{actpl}}}{\mathrm{d} \eta}=\mathfrak{M}_{\mathrm{act} \mathrm{pl}}\left[\phi_{\mathrm{th}}\right]\left[\phi_{\mathrm{pl}}\right] \\
& D_{\mathrm{th}} \frac{\mathrm{d}^{2} \phi_{\mathrm{th}}}{\mathrm{d} \eta^{2}}+3 \eta^{2} \frac{\mathrm{d} \phi_{\mathrm{th}}}{\mathrm{d} \eta}=0 \\
& \mathfrak{M}_{\mathrm{pl}}=\mathfrak{M}_{\mathrm{act} \mathrm{pl}}=\frac{k_{\mathrm{b}} L}{U_{\infty}} \frac{P e}{\beta^{2}(R)}
\end{aligned}
$$

The boundary condition for the numerical model are the same as those used in the analytical solution for $\eta=0$ and $\eta \rightarrow \infty$. A NAG (Ltd., N., 1999) routine (D02RAF) is used to solve the coupled set of o.d.e.'s [Eq. (28)] for a range of values of the reduced Damkohler number $\mathfrak{M}$. D02RAF solves the two-point boundary-value problem with general boundary conditions for a system of ordinary
TABLE III

\begin{tabular}{cccccccc}
\hline$K_{\mathrm{b}}\left(\mathrm{m} \mathrm{s}^{-1}\right)$ & $P e$ & $\kappa_{\mathrm{wt}}$ & $\tilde{D}_{\mathrm{th}}$ & $\kappa_{\mathrm{wp}}$ & $\tilde{D}_{\mathrm{pl}}$ & $\kappa_{\mathrm{wa}}$ & $\tilde{D}_{\text {act pl }}$ \\
\hline $5.0 \times 10^{6}$ & $1.0 \times 10^{6}$ & 0.1 & 10.0 & 1.0 & 1.0 & 2.0 & 1.0 \\
\hline
\end{tabular}

differential equations, using a deferred correction technique and Newton iteration. In this case, a continuation parameter was used that incremented the reduced Damkohler number $\mathfrak{M}$ from zero to the prescribed value. The routine also uses a mesh refinement technique that equidistributes an estimate of the truncation error across the mesh.

$\mathfrak{M}$ is a function of both the bulk reaction-rate $k_{\mathrm{b}}$ and the free-stream velocity $U_{\infty}$. Thus, variation in the source term for the conservation of species equation can come from either a variation in bulk reaction-rate or a change in $U_{\infty}$. It is noteworthy to investigate the relationship between the reduced Damkohler number and that of the free-stream velocity $U_{\infty}$. We can write

$$
\begin{aligned}
\mathfrak{M} & =\frac{k_{\mathrm{b}} L}{U_{\infty}}[P e]^{1 / 3} \frac{\left[9 \int_{0}^{R} \nu_{*} \mathrm{~d} R\right]^{2 / 3}}{\nu_{*}^{2}} \\
& =\frac{k_{\mathrm{b}} L}{U_{\infty}} \frac{\left[9 \int_{0}^{R} \nu_{*} \mathrm{~d} R\right]^{2 / 3}}{\nu_{*}^{2}}\left[\frac{U_{\infty}}{D L}\right]^{1 / 3} \propto U_{\infty}^{-2 / 3}
\end{aligned}
$$

Here $\nu_{*}$ is a wall slip velocity defined from the wall shear stress (see Appendix). From the above, it is seen that variations in the free-stream velocity $U_{\infty}$ may have a pronounced effect on $\mathfrak{M}$ and thus a considerable influence on the activation of platelets.

Reaction rates for the activation of platelets are not readily available in the literature, however, experimental evidence suggests that a figure of order $10^{6} \mathrm{~s}^{-1}$ (Strong et al., 1987) is not uncommon since the overall rate for the inhibition of thrombin by anti-thrombin III is of the same value. Using the value of $1.7 \times 10^{-13} \mathrm{~m}^{2} \mathrm{~s}^{-1}$ for the diffusion coefficient of platelets and, due to its size a value 10 times higher for thrombin, along with $L=0.003 \mathrm{~m}$; $U_{\infty}=0.01 \mathrm{~ms}^{-1}$; provides the characteristic Peclet number and Reynolds number for the flow conditions similar to those found in Affeld et al. (1995). It should be noted that Affeld et al.'s experiment had a value of $R e=2$. Adhesion rates from earlier mathematical models are available, however (Strong et al., 1987), Table III provides data used in the solution of both the analytical and numerical models.

\section{RESULTS}

Figure 4 shows platelet, activated platelet and thrombin concentrations for both the analytical and numerical solutions using the input data given by Table III and a reduced Damkohler number of $\mathfrak{M}=5.0 \times 10^{6}$. The root 

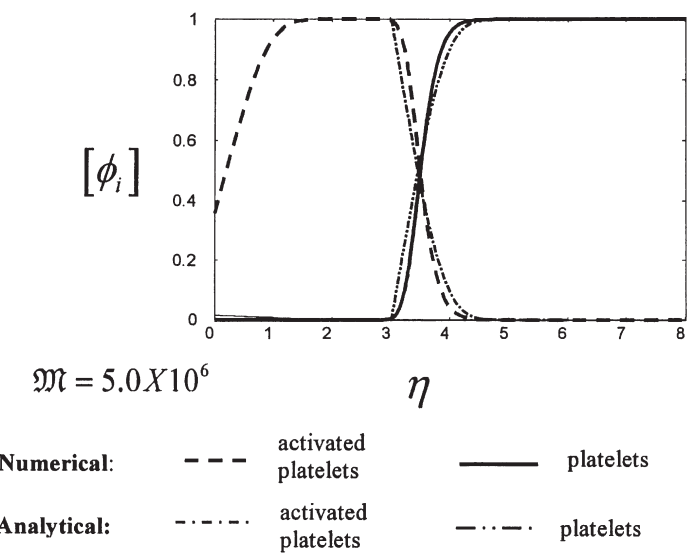

FIGURE 4 Analytical and numerical solutions for platelet, activated platelet and thrombin concentrations using the data of Table III and Damkohler number $\mathfrak{M}=5.0 \times 10^{6}$ as a function of the similarity variable $\eta$.

finding algorithm solution for Eq. (27) places the reaction sheet at $\eta=3$ for the cylindrical stagnation point system (accurate to three significant figures). Non-activated platelets diffuse toward the reaction sheet from $\eta>3$, whilst activated platelets diffuse from the reaction sheet toward the outer boundary. For values of smaller $\eta$, the concentration of activated platelets maintains a maximum value of 1 and then decreases towards the surface at $\eta=0$. The numerical solution shows that platelets are activated in a very small reaction zone, in this case in the neighbourhood of $\eta=3$, and this position separates the inner layer where, apart from very small concentrations of thrombin, only activated platelets exist and the outer layer where there are platelets and diffusing activated platelets. The thrombin concentration is small and is unaffected by the reaction since it is only a catalyst in the activation process.

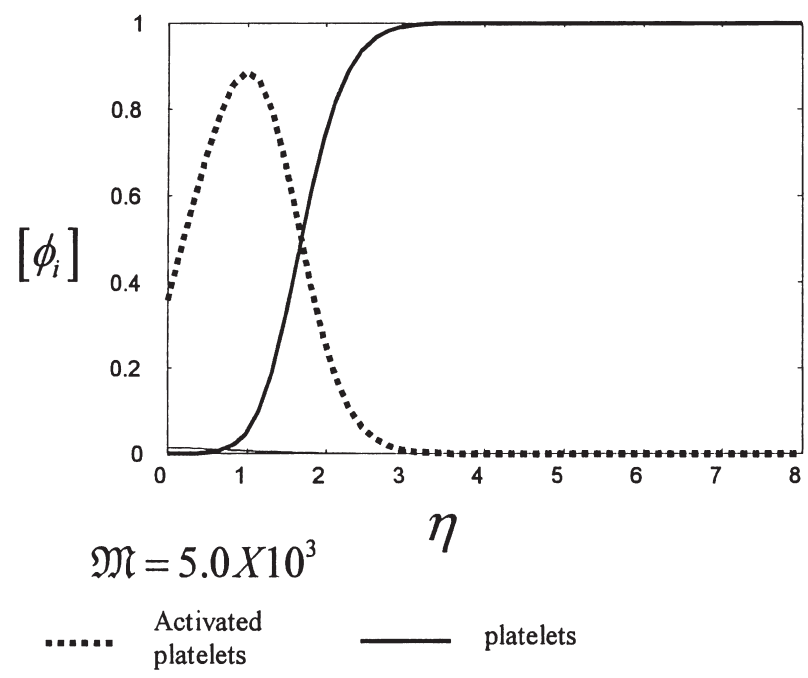

FIGURE 5 Concentrations of activated, non-activated platelets and thrombin versus similarity variable $\eta$ with $\mathfrak{M}=5.0 \times 10^{3}$ using the numerical solution.

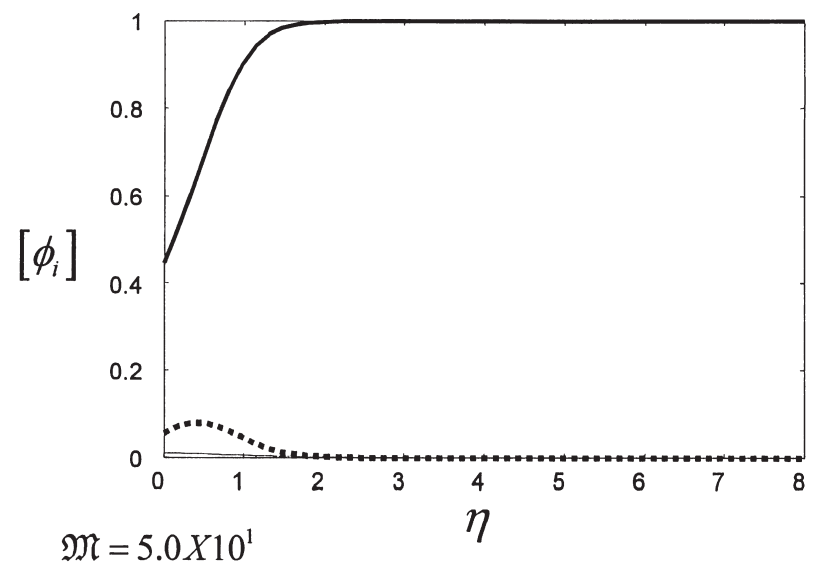

$\mathfrak{M}=5.0 \times 10^{1}$

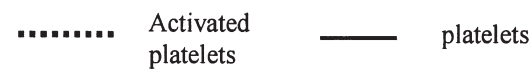

FIGURE 6 Concentrations of activated, non-activated platelets and thrombin versus similarity variable $\eta$ with $\mathfrak{M}=5.0 \times 10^{1}$ using the numerical solution.

When the Damkohler number is of intermediate size only the numerical solution can be used. From the results of the numerical model and using identical data to that of Fig. 4, apart from a reduction in $k_{\mathrm{b}}$ to allow a smaller reduced Damkohler number of $\mathfrak{M}=5.0 \times 10^{3}$, Fig. 5 shows the concentration of all three species as a function of the similarity variable $\eta$. The maximum concentration of activated platelets is now reduced, only $90 \%$ of that previously. In addition, the reaction zone is considerably larger, centred at a different position and occurring over the approximate range of $0.6<\eta<1.6$. The concentration of activated platelets at the adhesion surface is however similar to that for $\mathfrak{M}=5.0 \times 10^{6}$.

Figure 6 again shows platelet and activated platelet concentrations but this time with a Damkohler number (again by reducing $k_{\mathrm{b}}$ ) of $\mathfrak{M}=5.0 \times 10^{1}$. For this case the activated platelet concentration is a small perturbation to the non-reactive case. Almost no activated platelets are generated.

As an indicator of how thrombin production affects platelet adhesion, Fig. 7 illustrates the variation of the total platelet flux to the surface (for activated and non-activated platelets), $\dot{q}$, as a function of the rate of thrombin production at the adhesion surface per unit adhesion of platelets, $\kappa_{\mathrm{wt}}$. The value of $k_{\mathrm{b}}$ is now such that $\mathfrak{M}=5.0 \times 10^{6}$, as in the case presented by Fig. 4 . The total platelet flux to the wall is defined as

$$
\dot{q}=\left.\beta(R)\left(\frac{\mathrm{d} \phi_{\mathrm{act} \mathrm{pl}}}{\mathrm{d} \eta}+\frac{\mathrm{d} \phi_{\mathrm{pl}}}{\mathrm{d} \eta}\right)\right|_{\eta=0}
$$

As the thrombin production (per unit concentration of activated platelet), $\kappa_{\mathrm{wt}}$, increases, then the flux initially rises rapidly but tends to a constant value as $\kappa_{\mathrm{wt}} \rightarrow 3$. This plateau is possible due to the mass-transfer boundary layer being completely composed of activated platelets and hence saturated. 


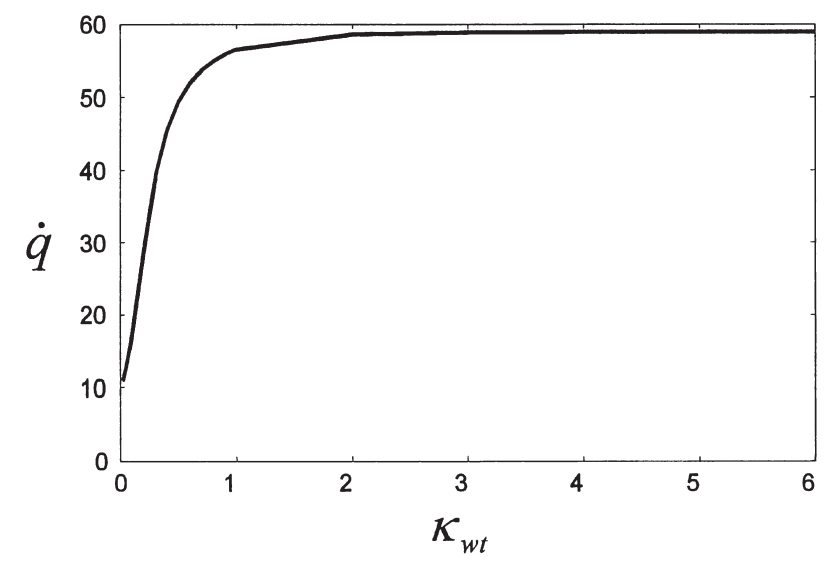

FIGURE 7 Platelet flux to the adhesive surface [Eq. (30)], evaluated at $R=0$ versus thrombin flux rate $k_{\mathrm{wt}}$ for $\mathfrak{M}=5.0 \times 10^{6}$.

It is important to investigate how the bulk reaction-rate affects the platelet adhesion at the surface. The variation of total platelet flux, $\dot{q}$, as a function of bulk reaction-rate, $k_{\mathrm{b}}$, is shown in Fig. 8. Here the bulk reaction-axis is logarithmic. In a similar manner to Fig. 7, the flux increases as the bulk reaction-rate increases until a plateau is reached for $k_{\mathrm{b}}>10^{6}$. Thus all variation of the platelet flux occurs over a range of approximately 100 . In this case the plateau corresponds to the situation modelled by the analytical solution given by Eqs. (22-26).

In the light of the variation of Damkohler number with both $k_{\mathrm{b}}$ and $U_{\infty}$, it is also necessary to look at the total platelet flux to the adhesion surface, $\dot{q}$, as a function of characteristic velocity $U_{\infty}$ and this is shown in Fig. 9. Two curves are plotted for values of the bulk reaction-rate $k_{\mathrm{b}}=4 \times 10^{5}$ and $4 \times 10^{7}$. For the case of $k_{\mathrm{b}}=4 \times 10^{5}$, the flux increases to a maximum value where $U_{\infty} \approx 0.6$. It then decreases rapidly over the range $0.6 \leq U_{\infty} \leq 2.0$. When the bulk reaction-rate is increased to $k_{\mathrm{b}}=4 \times 10^{7}$, then the platelet flux maximum is reached at $U_{\infty} \approx 2.0$. The shape of the curve is similar for both values of the bulk reaction-rate and it should be noted that for smaller values of $U_{\infty}$, the curves lie on top of each other. For lower

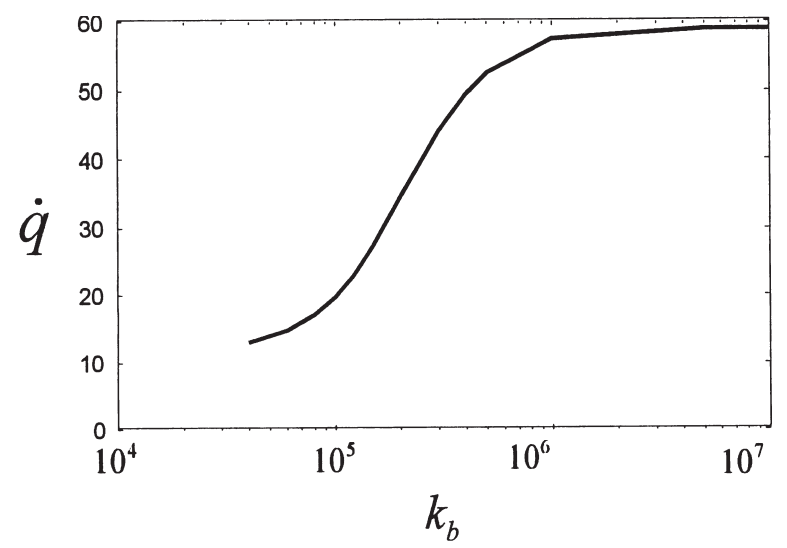

FIGURE 8 Platelet flux to the adhesive surface [Eq. (28)], evaluated at $R=0$ versus platelet activation rate $k_{\mathrm{b}}$ for $\mathfrak{M}=5.0 \times 10^{6}$.

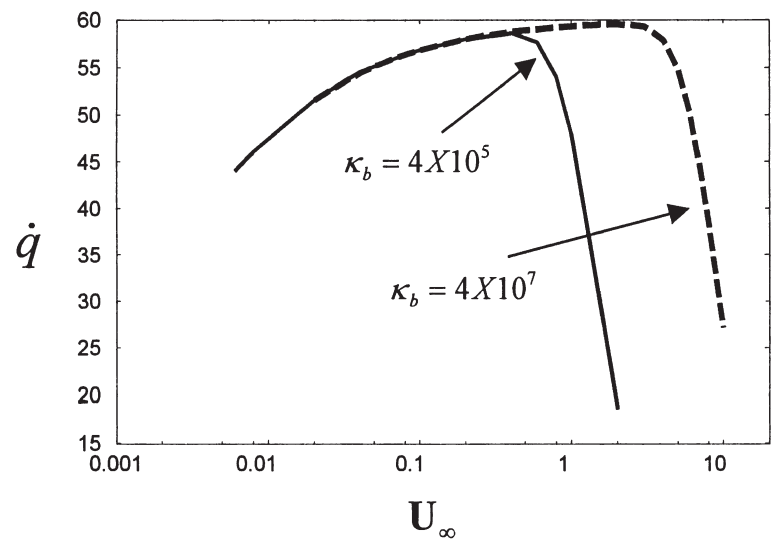

FIGURE 9 Platelet flux to the adhesive surface [Eq. (28)], evaluated at $R=0$ versus free-stream velocity $U_{\infty}$ for $k_{\mathrm{b}}=4 \times 10^{5}$ and $4 \times 10^{7}$.

but increasing values of $U_{\infty}$, more platelets are being convected into the mass-transfer boundary layer, however, as $U_{\infty}$ increases beyond a critical value, then the bulk reaction cannot be sustained and platelet activation decreases.

Figure 10 shows the calculated flux of platelets to the surface for the stagnation point flow case as a function of the stream-wise co-ordinate $R$. This shows clearly that the maximum flux occurs at the stagnation point streamline, in contrast to that found in the experimental data of both Affeld et al. (1995) and Karino and Goldsmith (1979). In addition, the platelet flux for a constant shear flow profile (Poiseuille flow), where the downstream co-ordinate is $x$, is also shown for comparison. For both cases, the flux is dominated by the shape of the function $\beta(R)$ or $\beta(x)$ [see Eqs. (12) and (14)].

For initial platelet adhesion, the steady-state solution may be used to estimate the number of platelets adhered to the surface at a certain time; this assumption has been used previously as shown by Strong et al. (1987).

Figure 11 shows the dimensional number of adhered platelets per unit area, as a function of axial distances as evaluated by Sorensen et al. (1999a) with parallel plate

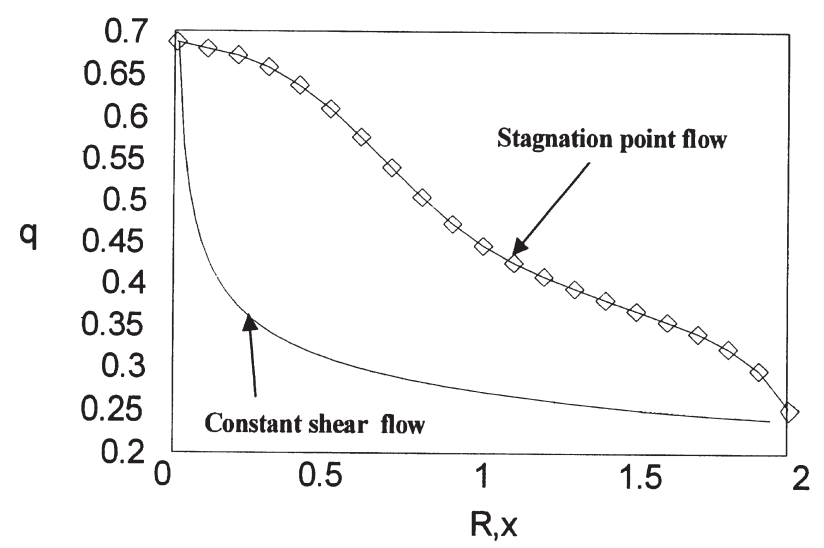

FIGURE 10 Platelet flux to the adhesive surface [Eq. (28)], versus nondimensional radial co-ordinate for the stagnation point flow and axial co-ordinate. 


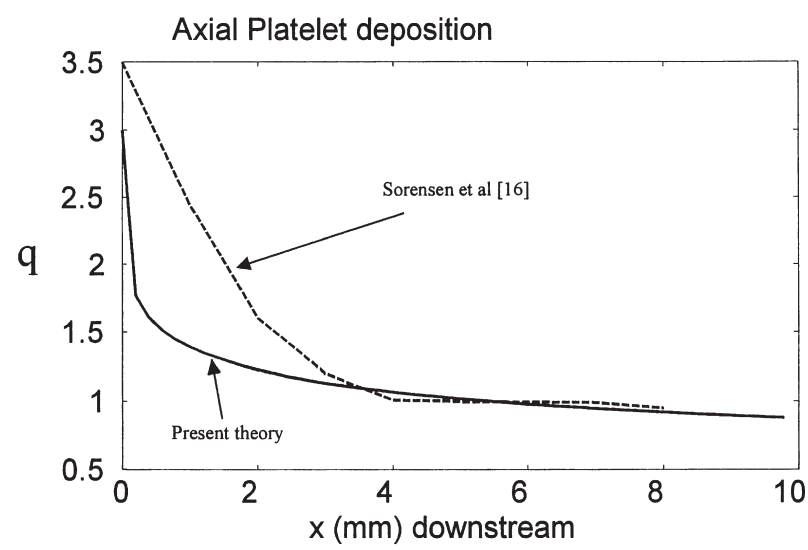

FIGURE 11 Number of adhered platelets per unit area, as a function of axial distance as evaluated by Sorensen et al. (1999a) with parallel-plate experiments (after $75 \mathrm{~s}$ ), compared with that calculated by the present theory.

experiments (after $75 \mathrm{~s}$ ), compared with that calculated by the present theory $(\beta(x)$ defined by Eq. (14)). By comparing these two models, it should be noted that the kinetic mechanism of Sorensen is considerably more complex, especially in the inhibition of thrombin in the bulk flow.

For values of the downstream co-ordinate $x>3$, the agreement is excellent. However, for smaller values of the downstream co-ordinate, the present theory predicts a smaller flux. Fogelson and Wang (1996) provided a model of thrombin production diffusing through the canaliculae of activated platelets. This can be modelled in a simple fashion by including a non-zero source term (proportional to the bulk concentration of activated platelets) in the conservation equation for thrombin [Eq. (28)]. The coefficient of proportionality is the same as that for the surface boundary condition for thrombin. Thus the activated platelets are a source for thrombin both in the bulk fluid and at the surface. Figure 12 shows the concentrations for platelets, activated platelets and thrombin (magnitude $\times 100$ ) for this case where the

$\left[\phi_{i}\right]$

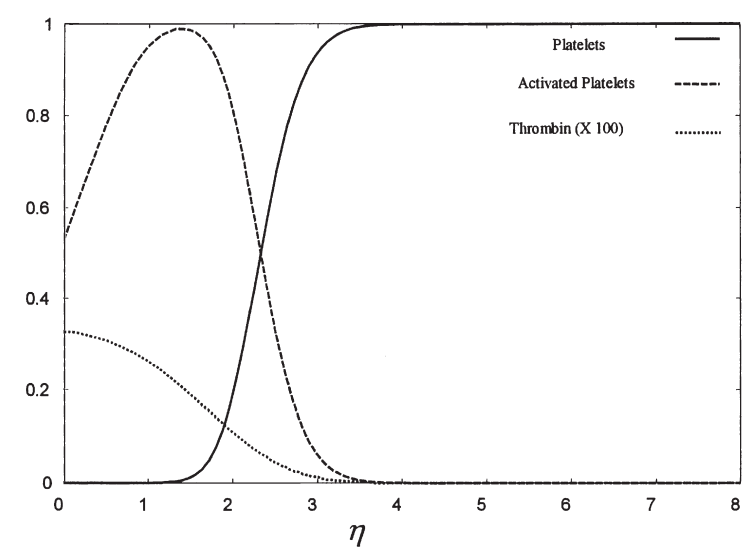

FIGURE 12 Concentrations for platelets, activated platelets and thrombin (magnitude $\times 100$ ) versus the similarity variable $\eta$, the input data is as that for Fig. 5 .

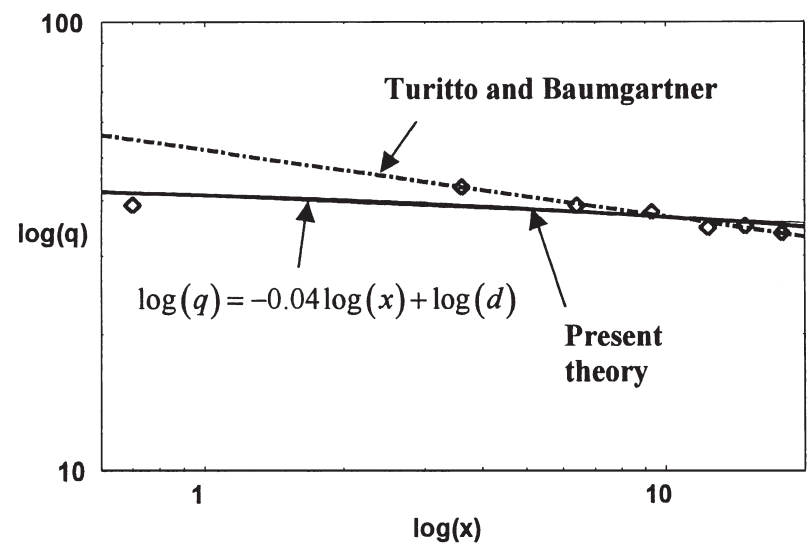

FIGURE 13 Log-Log plot of platelet flux versus axial distance compared with that of Turitto and Baumgartner (1975).

same input data has been used as that for Fig. 5. The activated platelets, in exuding thrombin, have increased the concentration of activated platelets and have moved the reaction zone further from the adhesion surface (compare with Fig. 5). If the production of thrombin per unit concentration of activated platelets is increased, then the reaction surface is moved even further away from the adhesion surface and the maximum concentration of activated platelets is increased.

On the basis of a power law relationship between shear rate and platelet diffusion, Turitto and Baumgartner (1975) plotted platelet adhesion data from parallel perfusion chamber experiments on $\log -\log$ axes. In doing so, they stated that the initial data point value was too low according to their theory and put this down to its axial position being "too close to the vessel edge." Their straight-line fit, therefore, did not use this data point. Figure 13 compares the present theory with the experimental data of Turitto and Baumgartner where their experiment used a simple flatplate perfusion chamber. Due to the possible uncertainty of the value of the diffusion coefficients, we can write the equation for platelet flux in the form

$$
q(x)=\frac{\kappa}{1+\kappa a x^{1 / 3}}
$$

and use a least squares Marquardt-Levenberg algorithm to fit to the experimental data of Turitto and Baumgartner's rate of platelet adhesion (Turitto and Baumgartner, 1975) via the parameters $a$ and $\kappa$. For this case, the parameter $a$ takes into account the constant shear stress found in a flatplate perfusion chamber experiments of Turitto and Baumgartner. Table IV shows values found for $a$ and $\kappa$.

TABLE IV

\begin{tabular}{lccl}
\hline Variable & Value & $63 \%$ conf. interval & \\
\hline$\kappa$ & 46.18 & \pm 3.74 & $\sim 8 \%$ \\
$a$ & 0.00257 & \pm 0.000911 & $\sim 35 \%$ \\
\hline
\end{tabular}


Figure 13 shows the data of Turitto and Baumgartner (1975) along with the least squares fit using Eq. (31). In addition, using the same type of analysis as that of Turitto and Baumgartner, Fig. 13 also shows a linear least squares fit of the form $\log (q)=m \log (x)+\log (c)$. The present theory shows a very good agreement with experiment.

\section{DISCUSSION}

The analytical solution for $D m \rightarrow \infty$ shows excellent agreement with that from the numerical model. In fact, for values of $\eta<3$, the curves lie on top of one another and similarly for $\eta>5$. Asymptotic analysis could be used to investigate the details of the reaction region when $\mathrm{Dm}$ is large, but not infinite. However, this may be counter productive since the region would certainly be of the order of the cell diameter for platelets.

The three different cases where the bulk reaction-rate is reduced, shown above in Figs. 4-6, indicate that as the Damkohler number is reduced, the reaction zone both widens and moves closer to the adhesion surface. Below a value of $k_{\mathrm{b}}=10^{5}$ the activation of platelets has virtually ceased and only non-activated platelets are adhering and present at the adhesion surface and this is a limiting condition. Whilst for large values of $k_{\mathrm{b}}$ the flux attains a plateau, again a limiting case, whose value is essentially controlled by the adhesion rate of activated platelets $\kappa_{\text {wa }}$.

The platelet flux as given by Eq. (30) is a function of the thrombin flux emanating from the adhered platelets and is shown in Fig. 7. Here, as $\kappa_{\mathrm{wt}}$ decreases and the flux of thrombin from the surface tends to zero, then the concentration of activated platelets reduces and the platelet flux tends to a limiting case. This is where cells diffusing toward the surface are only non-activated platelets in a manner similar to that of the platelets when no bulk reaction occurs. In contrast, as the value of $\kappa_{\mathrm{wt}}$ gets much larger than unity, then the reaction zone moves farther away from the surface and there exist essentially two layers. The inner layer, where only activated platelets exist, and the outer layer, where there are platelets yet to be activated. The activated platelets diffuse toward the adhesion surface from a maximum value of unity and the situation is similar to that found in Fig. 4. The outer layer consists of non-activated platelets diffusing toward the reaction zone, where their concentration is negligibly small, and activated platelets diffusing outward from the reaction zone. The resulting flux to the adhesion surface comes from the activated platelets only and tends to a limiting value as $\kappa_{\mathrm{wt}} \rightarrow \infty$ as shown later. The same condition arises for the case of the reaction rate of activation when $\kappa_{\mathrm{b}} \rightarrow \infty$ as shown in Fig. 8 . The inner layer is populated by activated platelets alone and the flux is determined almost entirely by the rate of adhesion at the surface. For the case of high or even moderate values of bulk reaction-rate, the activated platelet concentration behaves in a manner given by a simple convection diffusion equation, not unlike Eq. (19). Thus the concentration profile has the form at the surface given by

$$
\phi_{\text {act pl }}(0)=\frac{3 \tilde{D}_{\mathrm{actpl}} \beta(R)}{3 \beta(R)+\kappa_{\mathrm{wa}} \Gamma\left(1 / 3, \eta^{*}\right)}
$$

[derived from Eq. (22)] and the flux to the surface is

$$
\dot{q}=\frac{3 \kappa_{\mathrm{wa}} \tilde{D}_{\mathrm{actpl}} \beta(R)}{3 \beta(R)+\kappa_{\mathrm{wa}} \Gamma\left(1 / 3, \eta^{*}\right)}
$$

We note that $\Gamma\left(1 / 3, \eta^{*}\right)$ is not too dissimilar in value to $\Gamma(1 / 3, \infty)$. When the surface adhesion rate, $k_{\text {wa }}$, is high then the flux is given by

$$
\lim _{k_{\mathrm{wa}} \rightarrow \infty}(\dot{q})=\left(\frac{3 \tilde{D}_{\mathrm{actpl}} \beta(R)}{\Gamma\left(1 / 3, \eta^{*}\right)}\right)
$$

which is a constant for constant values of either $R$ or $x$ depending on whether the co-ordinate system is cylindrical or Cartesian, respectively. Equation (33) shows that for intermediate adhesion kinetics and high activation rate, then for there to be a significant reduction in the flux to the surface we must have that

$$
\begin{aligned}
1 & \sim \frac{\kappa_{\mathrm{wa}} \Gamma\left(1 / 3, \eta^{*}\right)}{3 \beta(R)} \Rightarrow \frac{3 \beta(R)}{\Gamma\left(1 / 3, \eta^{*}\right)} \\
& =\frac{3}{\Gamma\left(1 / 3, \eta^{*}\right)} \frac{\left\{\tau_{\mathrm{w}}(R)\right\}^{1 / 2}(P e R e)^{1 / 3}}{\left[9 \int_{0}^{R}\left\{\tau_{\mathrm{w}}(\gamma)\right\}^{1 / 2} \mathrm{~d} \gamma\right]^{1 / 3}} \approx \kappa_{\mathrm{wa}}
\end{aligned}
$$

The relationship between adhesion rate and fluid dynamics becomes a simple balance between $\beta(R)$ and $\kappa_{\mathrm{wa}}$. The convective and diffusive terms are taken into account by the similarity coefficient that shows the fundamental relationship between wall shear stress and the diffusion of platelets. This will be true for all 2D flow conditions provided the flow is fully attached or is in the neighbourhood of an attachment stagnation point (wall shear stress is well behaved) and is known a priori.

In contrast to the reduction in Damkohler number due to a reduction in bulk reaction rate $k_{\mathrm{b}}$, an increase in $U_{\infty}$, although providing a similar decrease in Damkohler number, can produce a maximum in the platelet flux. For this maximum to exist, there must be two competing effects. As the velocity $U_{\infty}$ decreases, $\beta(R)$ also decreases (for all $R$ or $x$ if a 2D system is being considered) and effectively reduces the platelet flux to the adhesion surface. However, the Damkohler number increases due to Eq. (28) and effectively strengthens the reaction source term whilst in contrast to moving the reaction zone away from the adhesion surface. The activated platelets have to diffuse further toward the surface and their concentration at the adhesion surface thereby reduces. As the velocity 
$U_{\infty}$ increases, the reaction surface moves toward the adhesion surface, but the Damkohler number decreases sufficiently to cause a significant decrease in the amount of activated platelets generated and the total platelet flux is reduced. As the bulk reaction-rate increases, the position of maximum platelet flux also increases.

If the characteristic length, $L$, is maintained constant, then Fig. 8 could be thought of as showing the relationship between platelet flux and a characteristic "global" shear rate given by $U_{\infty} / L$. For the stagnation point flow regime this ratio could be thought of as the "strength" of the stagnation flow. However, it should be noted that this "shear rate" is not a local one, but characteristic of the "experiment" as a whole. Experiments by Turitto et al. (1979), where the rate of platelet adhesion is plotted against "global" shear rate, also show this maximum behaviour for both rabbit and human blood. They stated that the reason for this maximum was, at the time, unclear. Although, it should be stated that the experiments were carried out with a sodium citrate anticoagulant that has the effect of inhibiting activation by thrombin.

In comparing the work of Sorensen et al. (1999a,b) with the present theory, we see that the more complex numerical kinetic mechanism of Sorensen predicts a larger number of platelets adhering to the upstream surface than that evaluated by the present model. There could be several reasons for this. Firstly, the Sorensen simulation is time dependent and the reactive surface boundary conditions take account of a reducing area available for adhesion. Initial studies with a time-dependent algorithm (David et al., 2001a) show that this is possibly due to axially dependent scaling factors multiplying the diffusive and convective terms for the time-dependent equation. A fuller investigation is left for a further paper. Secondly, the bulkreaction scheme takes into account the inhibition of thrombin using the work of Griffith $(1982 a, b)$. If this were incorporated into the present scheme, the first-order reaction rate for heparin and antithrombin III would increase as the platelets moved downstream.

In the present model, the activation of platelets has been taken into account using the reaction of thrombin with platelets where thrombin has emanated from activated and adhered platelets. In the work by Affeld et al. (1995) using a axially symmetric stagnation point flow, it was hypothesised that the thrombin would be convected downstream and "at some critical shear rate" induce a higher (maximum) value of platelet flux away from the stagnation point streamline, which had been observed in the experiments. The platelet flux to the surface is evaluated by Eq. (30). Consequently, the variation with downstream co-ordinate $R$ varies as $\beta(R)$. It has already been shown (David et al., 2001b) that $\beta(R)$ is a monotonic decreasing function for both stagnation point flow and for constant wall shear stress conditions. By inspection of Fig. 10, the maximum non-dimensionalised platelet flux occurs at the stagnation point streamline $(R=0)$, in contradiction to that found by experiments
(Reininger et al., 1993; Affeld et al., 1995; Reininger et al., 1996). Although it is recognised that RBCs can augment the diffusion of platelets, Affeld et al.'s experiments used platelet-rich plasma and hence contained no RBCs. Nor can the flux of thrombin emanating from the adhesion surface, assumed by Affeld, alone cause the particular adhesion distribution found. An additional phenomenon must be present for the variation in $R$, found by experiment, to occur. It has been shown (David et al., 2001b) that an adhesion rate, which, if defined as a function of shear rate, can provide the required variation, as suggested by Rajagopalan et al., 1988; Weiss, 1995). However, the comparison is not so good far downstream of the maximum wall shear stress. As in the case of Sorensen et al., this may be due to the time-dependency of the adhesion process and experiment.

Experiments by Vaishnav et al. (1983) on determining the erosion stress of endothelium under a stagnation point flow show a striking resemblance to the platelet-adhesion experiments of Affeld and may point the way to showing the relationship between the local shear stress and platelet adhesion. Platelet-adhesion reduction was also seen in the neighbourhood of the stagnation point by Karino and Goldsmith (1979).

For a stagnation point flow condition, the local shear rate does vary as mentioned earlier and this variation is taken into account by the similarity coefficient $\beta(R)$. This would also be the case for any 2D flow where the wall shear stress varies as a function of the stream-wise co-ordinate. Hence, for stagnation flows such as that exhibited by reattachment points downstream of stenotic vessels, which can be characterised with a certain strength, or any flow field where the wall shear stress varies, platelet adhesion may not occur at all if the reduced Damkohler number given by

$$
\mathfrak{M}=\frac{k_{\mathrm{b}} L}{U_{\infty}} \frac{P e}{\beta^{2}(R)}
$$

is decreased sufficiently. For the stagnation point flow case $\mathfrak{M}$ is a minimum at the stagnation point streamline and the bulk reaction mechanism is virtually extinguished.

The presented model has been used, with only a different shear stress substituted into the similarity variable definition (implicitly defined by the least squares fit), for comparison with the experiments of Turitto and Baumgartner (1975). This comparison shows that the model agrees very well with the experimental data. In fact, it predicts that the data point left out by Turitto and Baumgartner is a true representation of the adhesion mechanism and not a "rogue" point as assumed by Turitto and Baumgartner.

\section{CONCLUSIONS}

A mathematical model is presented for the convection, diffusion and reaction of platelets and thrombin. The local 
shear rate is incorporated into the representation by the definition of a similarity variable such that the model can allow for any a priori known 2D or axi-symmetric "wellbehaved" wall shear stress. The results show that for large values of the Damkohler number, which can occur for high platelet activation reaction or low free-stream velocity, there exist two layers. The inner layer consists of activated platelets that diffuse toward the adhesion surface and an outer layer consisting almost entirely of non-activated platelets. The layers are separated by the infinitesimally thin reaction zones where the platelets are activated. In this high Damkohler number case, the flux of platelets to the wall is dominated by the adhesion rate of activated platelets.

The mathematical model provides a simple relationship between this adhesion rate and the parameters of Peclet and Reynolds number for a range of kinetic conditions, reaction or diffusion controlled or intermediate.

As the Damkohler number decreases, the reaction zone broadens and moves toward the surface. If wall shear stress is assumed to be a function of free-stream velocity, then the model shows that, in accordance with the hypothesis set out by Caro et al. (1971), as the free-stream (characteristic) velocity increases, the bulk reaction decreases until no activation takes place and adhesion essentially stops. For this case, the convection of cells through the reaction site is so fast that activation, and hence adhesion, has little time to take place. Local areas of platelet adhesion may therefore occur for "advantageous" values of the local wall shear stress as this directly affects the reduced Damkohler number. Corresponding "in vivo scenarios" would be in the immediate downstream portion of stenosed vessels where slow recirculating flow exists as well as reattachment stagnation points. Similar situations arise in the sinus region of the aortic root when prosthetic valves are in place, especially if insufficient washout occurs.

In varying the free-stream velocity, the platelet adhesion flux to the surface attains a maximum value. The freestream velocity value at which this occurs varies with the platelet activation reaction rate.

Comparison with the parallel-plate perfusion model of Sorensen et al. (1999a,b) shows that the present model predicts a lower platelet flux close to the start of the reactive surface. However, this is probably due to the presented model being of a steady-state form. Earlier work has shown that if time-dependency is taken into account the convective and diffusive terms have a stream-wise scaling factor that provides for a higher platelet adhesion at low values of the stream-wise co-ordinate.

The presented model representing both adhesion and the presence of the activating species thrombin emanating from either the adhered platelets or non-adhered activated platelets does not provide the required variation in platelet flux for a stagnation point flow compared to that seen in experiments. It is probable that another factor, possibly a shear-rate dependent adhesion reaction-rate, is in play in contrast to the "critical shear rate" theory proposed by Affeld et al. (1995).

\section{Acknowledgements}

We would like to acknowledge the kind support of the British Council and the Netherlands organisation for Scientific Research (NWO).

\section{References}

Affeld, K., Reininger, A.J., Gadischke, J., Grunert, K., Schmidt, S. and Thiele, F. (1995) "Fluid mechanics of the stagnation point flow chamber and its platelet deposition", Artif. Organs 19(7), 597-602.

Caro, C.G., Fitz-Gerald, J.M. and Schroter, R.C. (1971) "Atheroma and arterial wall shear. Observation. Correlation and proposal of a shear dependent mass transfer mechanism for atherogenesis", Proc. R. Soc., Lond. Ser. B 177, 109-159.

David, T., Thomas, S., Walker, P.G., et al. (2001a) "Models of platelet deposition in stagnation point flow", In: Middleton, J., ed, Computer Methods in Biomechanics and Biomedical Engineering-3 (Gordon and Breach, Reading), pp 743-748.

David, T., Thomas, S. and Walker, P.G. (2001b) "Platelet deposition in stagnation point flow: an analytical and computational simulation", Med. Eng. Phys. 23(5), 299-312.

Fogelson, A.L. and Wang, N.-T. (1996) "Platelet dense-granule centralisation and the persistence of ADP secretion", Am. J. Physiol. 270(Heart Circ. Physiol., 39), H1131-H1140.

Friedman, L. and Leonard, E. (1971) "Platelet adhesion to artificial surfaces: consequences of flow, exposure time, blood condition and surface nature", Fed. Proc. 30(5), 1641-1648.

Griffith, M.J. (1982a) "The heparin enhanced antithrombin III/thrombin reaction is saturable with respect to both thrombin and antithrombin III", J. Biol. Chem. 257, 13899-13902.

Griffith, M.J. (1982b) "Kinetics of the heparin-enhanced antithrombin III/thrombin reaction. Evidence for a template model for the mechanism of action of heparin", J. Biol. Chem. 257, 7360-7365.

Hubbell, J.A. and McIntire, L.V. (1986) "Platelet active concentration profiles near growing thrombi. A mathematical consideration", Biophys. J. 50(5), 937-945.

Karino, T. and Goldsmith, H.L. (1979) "Adhesion of human platelets to collagen on the walls distal to a tubular expansion", Microvasc. Res. 17, 238-262.

Kratzer, M.A. and Kinder, J. (1986) "Streamline pattern and velocity components of flow in a model of a branching coronary vesselpossible functional implication for the development of localized platelet deposition in vitro", Microvasc. Res. 31(2), 250-265.

Leonard, E.F., Grabowski, E.F. and Turitto, V.T. (1972) "The role of convection and diffusion on platelet adhesion and aggregation", Ann. N.Y. Acad. Sci. 201, 329-342.

NAG Ltd. (1999) NAG Fortran Library Routine D02RAF.

Petschek, H., Adamis, D. and Kantrowitz, A.R. (1968) "Stagnation flow thrombus formation", Trans. Am. Soc. Artif. Internal Organs 14, $256-260$.

Rajagopalan, S., McIntire, L., Hall, E.R. and Wu, K.K. (1988) "The stimulation of arachidonic acid metabolism in human platelets by hydrodynamic stresses", Biochem. Biophys. Acta 958, 108-115.

Reininger, A.J., Reininger, C.B. and Wurzinger, L.J. (1993) "The influence of fluid dynamics upon adhesion of ADP-stimulated human platelets to endothelial cells", Thromb. Res. 71(3), 245-249.

Reininger, C.B., Graf, J., Reininger, A.J., Spannagl, M., Steckmeier, B. and Schweiberer, L. (1996) "Increased platelet and coagulatory activity indicate ongoing thrombogenesis in peripheral arterial disease", Thromb. Res. 82, 523-532.

Schlichting, H. (1960) Boundary Layer Theory (McGraw-Hill, New York).

Schoephoerster, R.T., Oynes, F., Nunez, G., Kapadvanjwala, M. and Dewanjee, M.K. (1993) "Effects of local geometry and fluid dynamics on regional platelet deposition on artificial surfaces", Arterioscler. Thromb. 13(12), 1806-1813.

Sorensen, E.N., Burgreen, G.W., Wagner, W.R. and Antaki, J.F. (1999a) "Computational simulation of platelet deposition and activation: II. 
Results for Poiseuille flow over collagen", Ann. Biomed. Eng. 27, $449-458$

Sorensen, E.N., Burgreen, G.W., Wagner, W.R. and Antaki, J.F. (1999b)

"Computational simulation of platelet deposition and activation: I. Model development and properties", Ann. Biomed. Eng. 27, $436-448$.

Strong, A.B., Stubley, G.D., Chang, G., Absolom, D.R. (1987) "Theoretical and experimental analysis of cellular adhesion to polymer surfaces", J. Biomed. Mater. Res. 21, 1039-1055.

Thomas, S.J. (2000) "The role of flow in thrombogenesis" PhD Thesis, School of Mechanical Engineering, University of Leeds (Leeds, UK).

Tippe, A., Reininger, A., Reininger, C. and Riess, R. (1992) "A method for quantitative determination of flow induced human platelet adhesion and aggregation", Thromb. Res. 67(4), 407-418.

Turitto, V. and Baumgartner, H. (1975) "Platelet deposition on subendothelium exposed to flowing blood: mathematical analysis of physical parameters", Trans. Am. Soc. Artif. Internal Organs 21, $593-601$.

Turitto, V., Weiss, H.J. and Baumgartner, H. (1979) "Rheological factors influencing platelet interaction with vessel surfaces", J. Rheol. 26, $735-749$

Vaishnav, R.N., et al. (1983) "Determination of the local erosion stress of the canine endothelium using a jet impingement method", J. Biomech. Eng. 105, 77-83.

Weiss, H.J. (1995) "Flow-related platelet deposition on subendothelium", Thromb. Haemostasis 74(1), 117-122.

Wilson, J.M. and Ferguson, J.J. (1999) "Platelet-endothelial interactions in atherothrombotic disease: therapeutic implications", Clin. Cardiol. 22, 687-698.

\section{APPENDIX}

\section{TRANSFORMATION OF THE CONSERVATION INTO THE O.D.E. BY SIMILARITY VARIABLE}

For brevity we look at only the cylindrical case in detail. The non-dimensionalised conservation equation for the platelet mass fraction can be written as

$$
u \frac{\partial \phi_{\mathrm{pl}}}{\partial R}+v \frac{\partial \phi_{\mathrm{pl}}}{\partial z}=\frac{1}{P e} \frac{\partial^{2} \phi_{\mathrm{pl}}}{\partial z^{2}}
$$

The velocity profile is assumed to be of a linear form since the ratio of mass-transfer boundary-layer thickness to the viscous boundary-layer thickness is small. A streamfunction $\psi$ is thus defined as

$$
\tilde{\psi}(R, z)=\frac{\rho}{2} \frac{\tilde{v}_{*}^{2}}{\mu} \tilde{z}^{2}
$$

where $v_{*}$ is the "friction" velocity defined by

$$
\tilde{v}_{*}=\left\{\frac{\tilde{\tau}_{\mathrm{w}}(R)}{\rho}\right\}^{1 / 2}
$$

$\tilde{\tau}_{\mathrm{w}}(R)$, a wall shear stress function whose values are known a priori. It should be noted that for the axisymmetric case the stream-function given by Eq. (A2) is not the normal Stokes stream-function. If $\tilde{\tau}_{\mathrm{w}}(R)$ is nondimensionalised by $\rho U_{\infty}^{2}$ so that

$$
v_{*}^{2}=\tau_{\mathrm{w}}(R)
$$

then the non-dimensional velocity components can then be written as

$$
\begin{aligned}
& u=v_{*}^{2} z \frac{\rho U_{\infty} L}{\mu}=v_{*}^{2} z R e \\
& v=-\frac{\rho}{2} \frac{\tilde{z}^{2}}{\mu} \frac{\mathrm{d}\left(v_{*}^{2}\right)}{\mathrm{d} \tilde{R}}=-\frac{z^{2}}{2} \frac{\mathrm{d}\left(\tilde{v}_{*}^{2}\right)}{\mathrm{d} R} R e
\end{aligned}
$$

We define two new variables thus,

$$
\xi=R ; \quad \zeta=v_{*} z R e
$$

and substituting the velocity expressions into the convective part of the conservation equation we have

$$
\begin{aligned}
u \frac{\partial \phi_{\mathrm{pl}}}{\partial R}+v \frac{\partial \phi_{\mathrm{pl}}}{\partial z} & =v_{*}^{2} z \operatorname{Re} \frac{\partial \phi_{\mathrm{pl}}}{\partial \xi}=\frac{R e^{2}}{P e} v_{*}^{2} \frac{\partial^{2} \phi_{\mathrm{pl}}}{\partial \zeta^{2}} \\
& \Rightarrow \frac{\zeta}{v_{*}} \frac{\partial \phi_{\mathrm{pl}}}{\partial \xi}=\frac{R e^{2}}{P e} \frac{\partial^{2} \phi_{\mathrm{pl}}}{\partial \zeta^{2}}
\end{aligned}
$$

By transforming again through the variables

$$
\begin{aligned}
& \sigma=\zeta\left(\frac{P e}{R e^{2}}\right)^{1 / 3} \\
& \chi=\int_{\xi_{0}}^{\xi} v_{*} \mathrm{~d} \xi
\end{aligned}
$$

and

$$
\eta=\frac{\sigma}{(9 \chi)^{1 / 3}}
$$

Upon substituting into Eq. (A6) we have, with a little algebra,

$$
\frac{\mathrm{d}^{2} \phi_{\mathrm{pl}}}{\mathrm{d} \eta^{2}}+3 \eta^{2} \frac{\mathrm{d} \phi_{\mathrm{pl}}}{\mathrm{d} \eta}=0
$$

the similarity variable $\eta$ can be written now in terms of the initial variables thus,

$$
\eta=z \frac{\left\{\tau_{\mathrm{w}}(R)\right\}^{1 / 2}(R e P e)^{1 / 3}}{\left[9 \int_{0}^{R}\left\{\tau_{\mathrm{w}}(\zeta)\right\}^{1 / 2} \mathrm{~d} \zeta\right]^{1 / 3}}=z \beta(R)
$$

Using a stream-function given by

$$
\tilde{\psi}(\tilde{x}, \tilde{y})=\frac{\rho}{2} \frac{v_{*}^{2}(\tilde{x})}{\mu} \tilde{y}^{2}
$$

a similar method provides the similarity variable for the Cartesian system. 


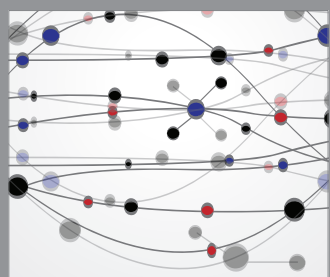

The Scientific World Journal
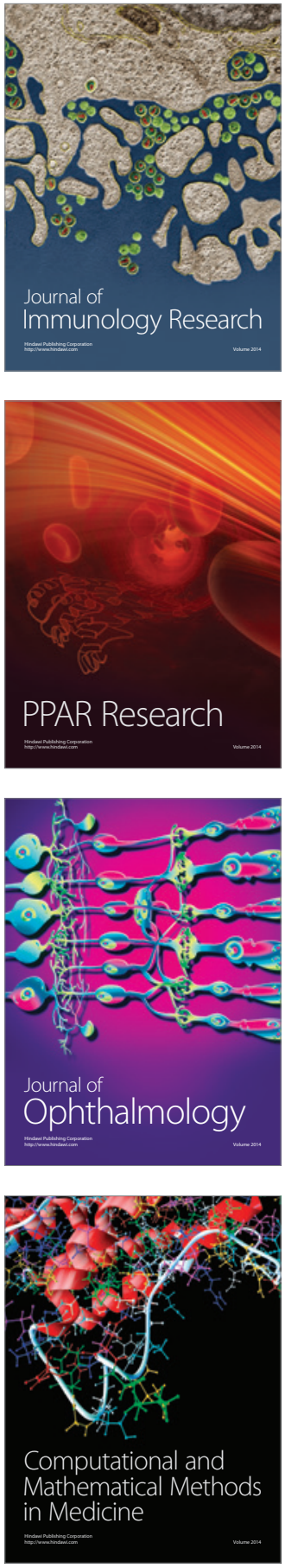

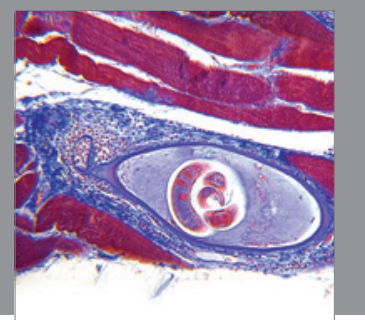

Gastroenterology

Research and Practice
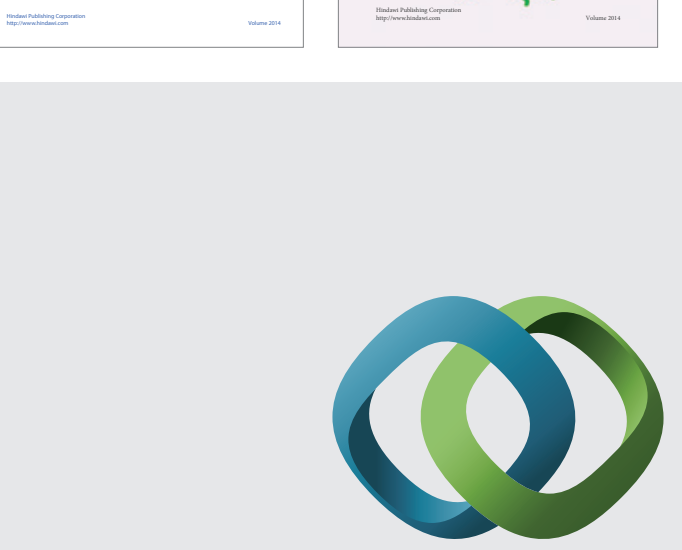

\section{Hindawi}

Submit your manuscripts at

http://www.hindawi.com
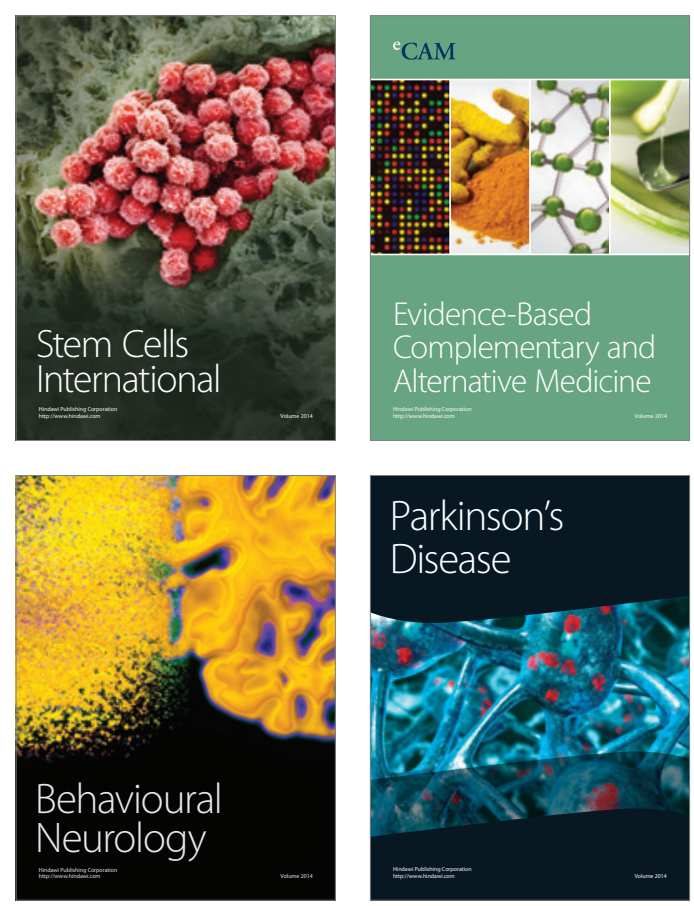

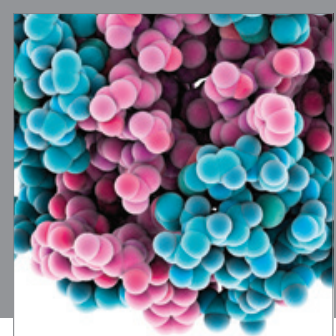

Journal of
Diabetes Research

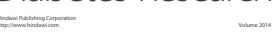

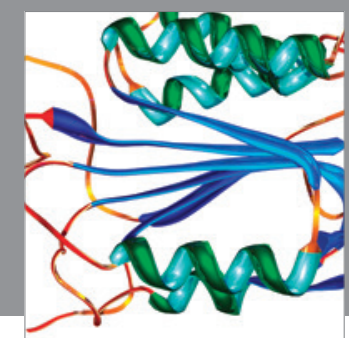

Disease Markers
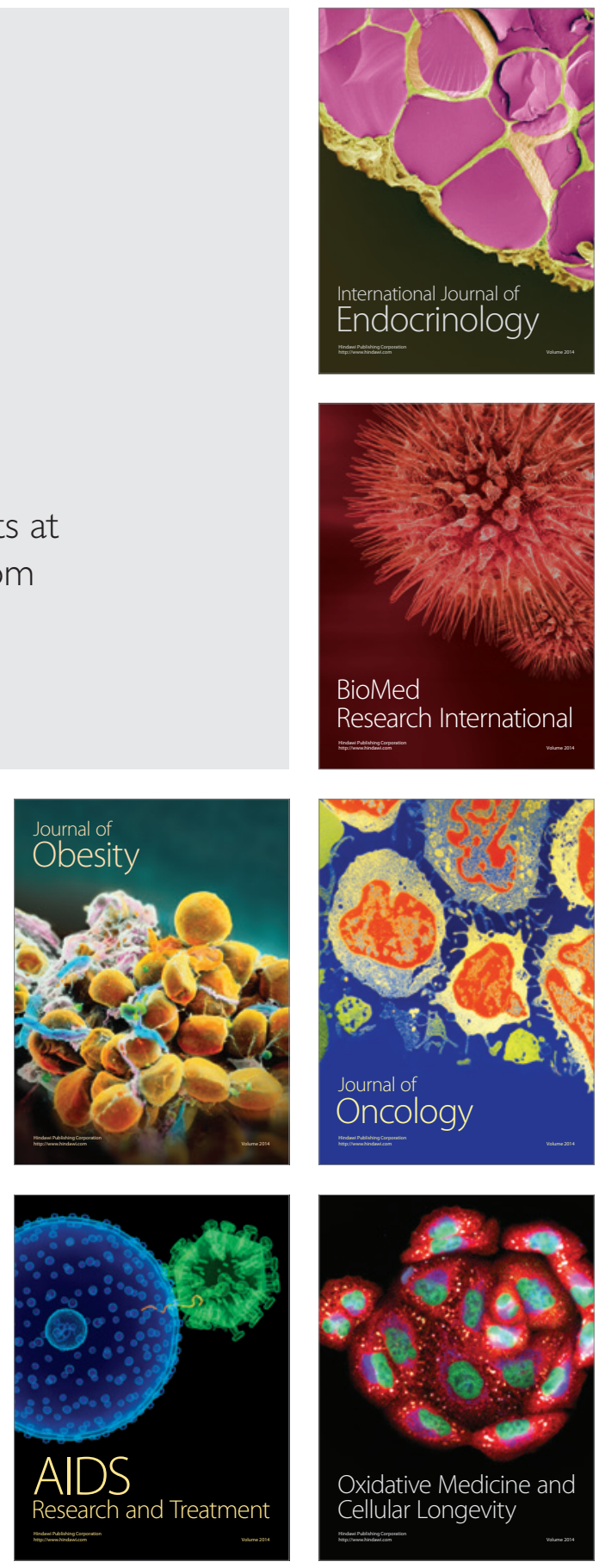